\title{
Adaptive Architecture: Towards Resiliency in the Built Environment
}

\author{
VERA PARLAC \\ University of Calgary
}

This paper discusses possibilities afforded by an integrative approach in which overlapping of intelligence, material capabilities, and social and ecological issues inspires an entirely new approach to designing resilience through adaptability. The ability to regulate behavior and adapt to the demands of a situation has always been associated with living organisms. This capacity to adapt is what defines resilience in nature. A technologically augmented built environment can often adapt to changes in its environment, but this adaptivity is often prescribed. If resilience is the capacity to recover from a disturbance and a traumatic event, how is then resilience manifested within a technologically enhanced setting? How do we design resilience into our engineered ecologies? How is this manifested in the design context where boundary between self developing and externally designed is increasingly blurred?

\section{INTRODUCTION}

The term resilience suggests certain immunity to trauma, an ability to recover quickly from an unexpected traumatic event. Synonyms for resilience, if related to a person, are tough, strong or hardy, or, flexible, pliable, or supple if related to an object. What is generally expected from a resilient built environment is a quick return to its prior condition and unchanged appearance. The fact that we expect a static response (status quo) from anything or any environment under the strain speaks about our attitude towards change and our tolerance (or the lack of it) for the unexpected. Even though, to be resilient, a thing needs to appear unchanged, it is also possible that a resilient thing is always altered. I start from this condition of being altered and propose it as a more viable starting point for the discussion of resiliency. To be resilient, the built environment should continue to operate and perform even if altered and preferably continue to alter itself as it 'rebuilds'. It is the process of alteration, transformation and change that should be 'designed' and channeled. To explore resiliency we should focus on dynamics and change and not on stasis and permanency.

In nature, the capacity of organisms to adapt to changes in their environment defines their resilience. In the built environment, resilience is traditionally seen as a capacity to resist a potentially catastrophic event and is achieved through the use of specific materials, specific construction techniques, or by engineering our way into a comfortable environment - in other words by strength or by 'more'. This attitude is very well reflected in Frei Otto's observation that "Architecture is man's oldest skill in his struggle for survival in nature. It is therefore directed against nature." ${ }^{11}$ But, what might make architecture truly resilient is its ability to productively participate within its larger ecology - to be given an opportunity to 'behave' according to a challenge it is facing. To achieve that, architecture and the built environment in general should be more tightly bound to the dynamics of local ecologies. Strong links to the undercurrents of the surroundings (near and far) could facilitate an active response to disruptions and could accommodate unexpected changes. In other words, adaptability and responsiveness could be key to resiliency in architecture.

Technological resilience in architecture could be achieved by incorporating directly into the built environment embedded and sensing technologies, data and energy harvesting, synthetic biology, robotics and/or material innovation. These technologies would make the built environment active and sensing - and not passive, as is presently the case. Such resiliency would consist of a capacity to anticipate and respond to changing environmental, programmatic or energy demands, and to actively engage the constructed fabric even in non-catastrophic events that might require some kind of adaptation.

Technologically augmented environments would interface with their larger contexts more productively because they are not inert, because they sense and communicate and more effectively extend into their surroundings. Technological resilience requires adaptability and responsiveness that in turn requires existence of (1) a boundary that could facilitate that response (as an interface) and (2) incorporation of technologies necessary to make otherwise inert environments active. Technological resilience would ideally result in the capacity of the built environment to retain its functions in spite of a strain (natural or man-made). Furthermore, when made of active and sensing material systems, environments can be closely linked to their local conditions and might be able to signal an unanticipated event long before it causes a problem.

In "The Thousand Dreams of Stelavista" James Graham Ballard describes "psychotropic" houses, constructed from a material he called plastex, that can bond with their inhabitants, sense their emotions and needs and adapt to them. ${ }^{2}$ We don't have plastex but the latest advances in distributed computation, embedded computing, sensing technologies (including brain wave sensing), biosensors, material innovation and synthetic biology (all coupled with digital design) are enabling proposals for integrated strategies that facilitate further development of adaptation and resilience in the built environment. 


\section{EXPLORING RESILIENCY}

As the natural and constructed worlds meet there is a need to control the boundary (interface) between them by preventing what we perceive to be undesirable effects. That very space is what generates some of the most interesting questions pertaining to the humanity's relationship to technology. Impermeability of a boundary that separates the constructed environments from the natural, and perception of what undesirable is, might need a second look.

This issue of the boundaries between the built and the natural were explored in student projects completed in a senior level research studio I taught at the University of Calgary. The studio explored in general the idea of responsiveness in architecture. It engaged questions of how a technologically augmented and resilient built environment can be designed and how it could participate in a larger ecology. We were interested in new forms of resiliency that are linked to their local ecologies (natural or constructed) and in articulation of innovative design approaches that integrate data, sensors, synthetic biology, or new materials. Students were encouraged to think in terms of flow and exchange of information, energy and matter rather than in terms of architectural form. The matter (material systems) was viewed as dynamic and active and a form understood as changing. Resulting projects demonstrated how technology could empower architecture to operate as an intelligent interface that connects spaces, users, performance criteria and environment in real time. The projects looked for more productive as well as creative ways to negotiate the boundary between the natural and the constructed by relying on some of the latest technological and scientific propositions. By focusing on technologically augmented environments that respond to spatial, programmatic or environmental pressures instead of an architectural object, the projects challenge conventional definitions of architecture. They underline a necessity to think of design space as dynamic and to incorporate change over time into the design proposals.

More specifically, the projects engaged the question of a permeable boundary (interface) by designing responsive spatial boundaries (see the Swarm Space project), programmatically or environmentally responsive modular systems (Augmentum and Remedia[c]tion), resilient dwellings (The Imminent Emergency Defense System), or explored the nature of a productive relationship with the larger ecology (Urban Reef, Charged Landscapes).

Swarm intelligence is at the core of the Swarm Space project (Figure 1) by Bin Tian. The project explores an application of a swarm intelligence algorithm as a way to negotiate between natural and artificial systems. It proposes a variable spatial boundary that responds to the idiosyncratic movement of people (collective or individual) and realigns the space to allow for adequate room size and the passage of people. The
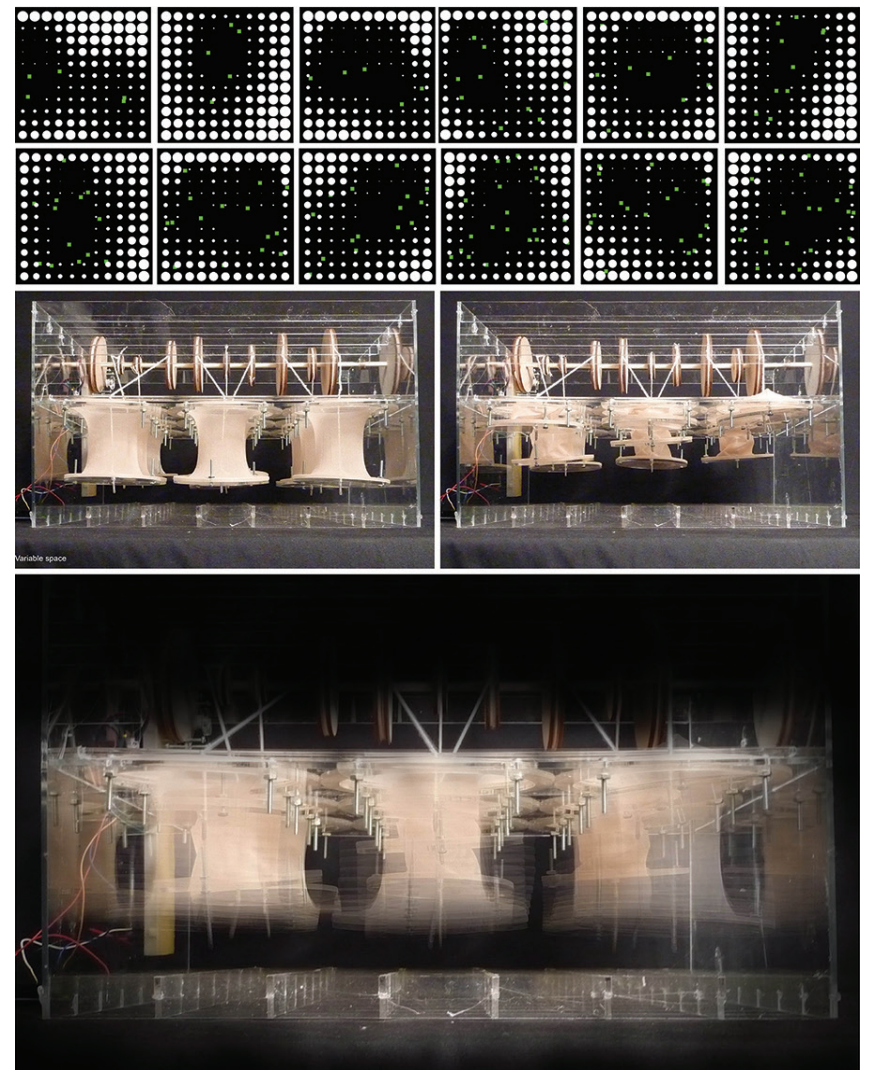

Figure 1 Above: Swarm Space by Bin Tian

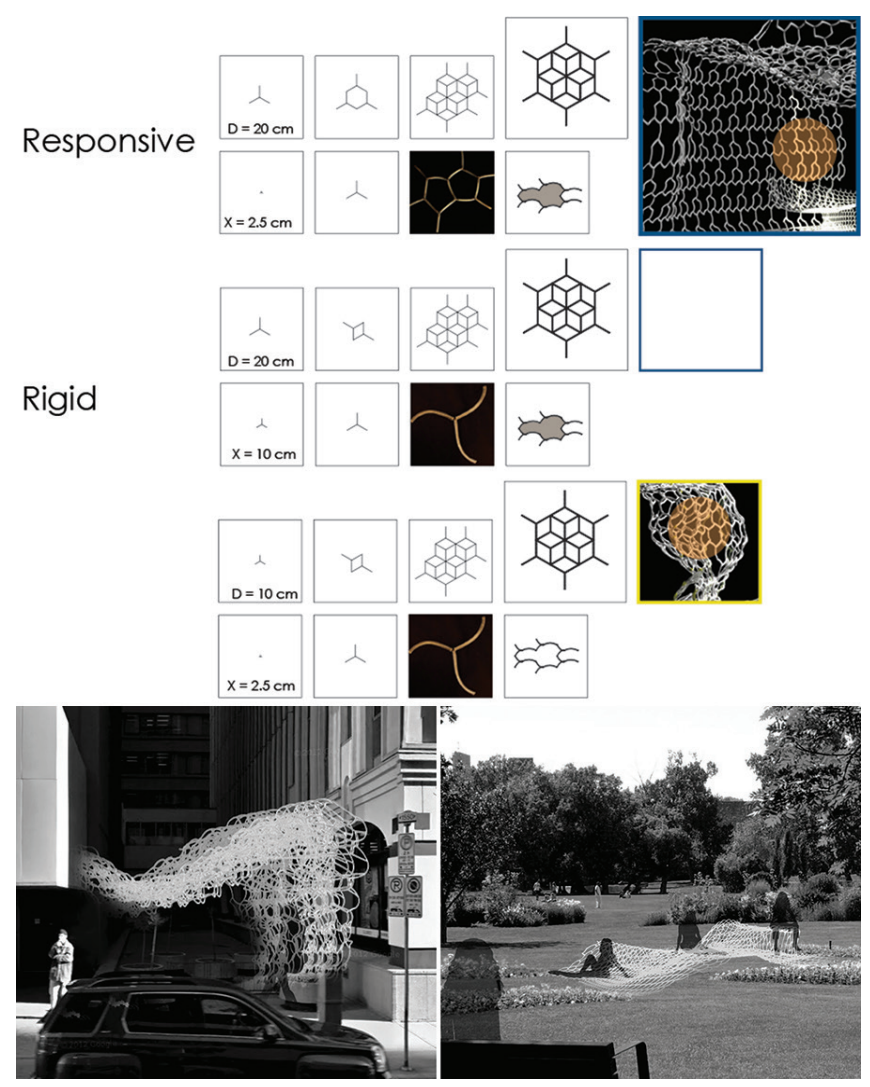

Figure 2: Augmentum by Faria Hamidzadeh 

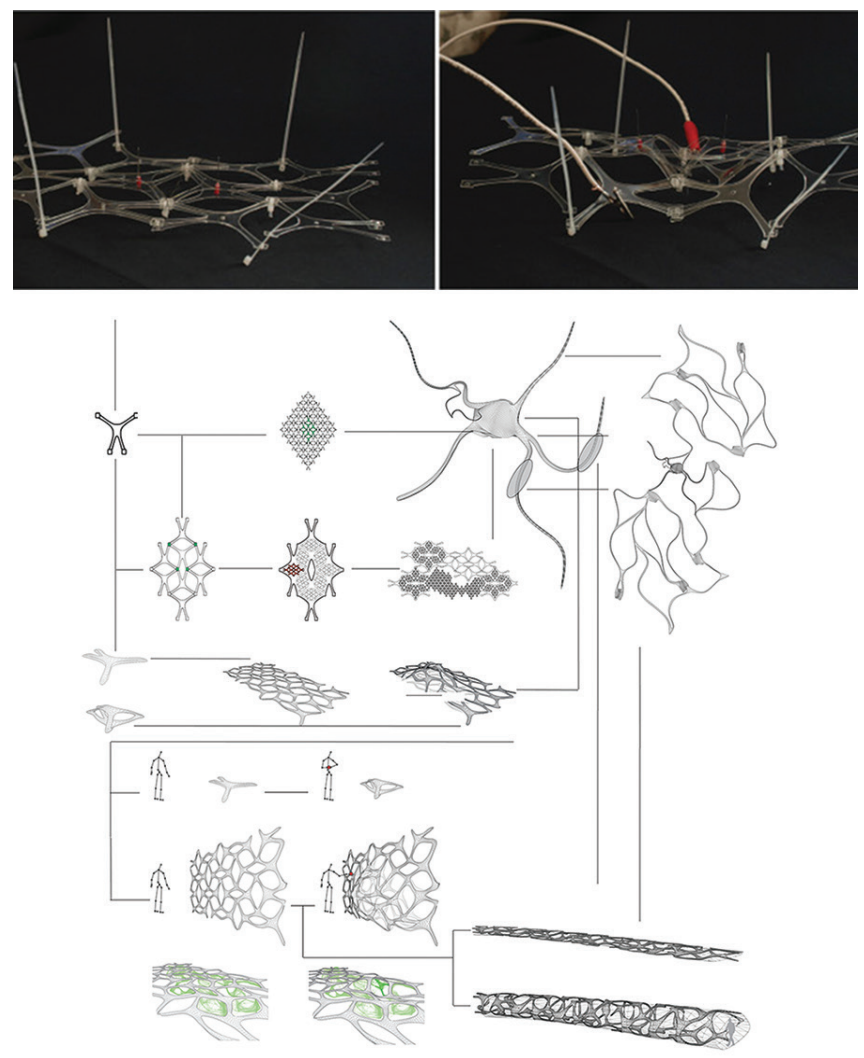

Figure 3 Above: Remedia[c]tion by Matt Parker.

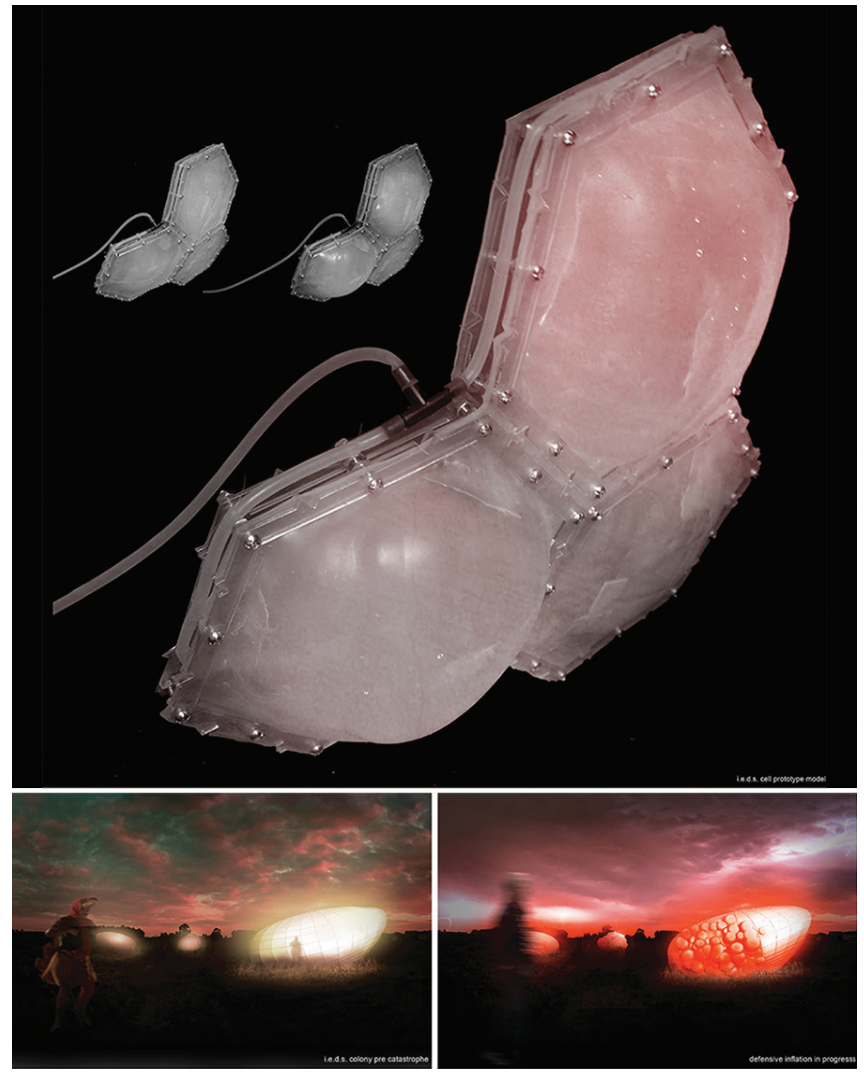

Figure 4: The Imminent Emergency Defense System by Kevin Spaans proposal offers a dynamic environment that addresses programmatic needs in real time. At the same time, the project explores the notion of a permeable boundary by offering a space divider whereby permeability is manifested in its changeability and capacity to offer varying degrees of passage (of people or light) or transformation (of space).

The projects Augmentum and Remedia[c]tion are based on self-similar modular and adaptive components. The Augmentum (Figure 2), by Faria Hamidzadeh, is an adaptive material system capable of adjusting to a wide variety of spatial conditions. It can be constructed within any public space and can serve as a transition between sheltered and open environments. It is easily erected out of a large number of self-similar components designed to enable the "growth" of the system, regardless of the spatial boundaries. By combining the components in a particular way, the system can vary from "soft" to "hard" to provide soft surface conditions as well as hard structural regions. The Augmentum can shift between parasitic and self-supporting structure and be constructed in a variety of urban void conditions. The cells of the structure have active or inactive infill. Active infill enables an energy-harvesting capacity that can power regions of the structure. Inactive cell infill provides a seating surface or shading. The project can be "grown" into a light sculpture, a landscape piece, an active façade, a shelter or a seat.

Remedia[c]tion (Figure 3) by Matt Parker, is a transportable aquatic disaster relief laboratory (and dwelling if needed) for a deep water oil spill remediation. It consists of a network of flexible pods that can be deployed to help stabilize and clean polluted marine ecosystems. The pods could be connected and reconnected to form variable configurations as needed. The project explores what it means for architecture to be fully integrated through a responsive system capable of sensing and productively adapting to environmental inputs and inhabitant occupation. The responsiveness of the system is reflected in its capacity to sense the level of pollution and to grow, cultivate, and release oil-degrading microbes as needed. Their production is located in the pod's "tentacles" and certain regions of its surface.

Both Augmentum and Remedia[c]tion explore the notion of an active material system as a way to interface with the surroundings and respond to their condition. The Augmentum supports spatial adaptability and energy requirements by exploring the idea of the 'growth' of the structure from selfsimilar components, while in the Remedia[c]tion the active material system is an operative boundary between the polluted natural environment and a constructed system that is actively participating in its remediation.

The Imminent Emergency Defense System (IEDS) project (Figure 4) by Kevin Spaans explores the idea of a disasterresilient dwelling. It proposes a living pod that reverses 
inevitable destruction expected of traditionally built houses in regions prone to major meteorological catastrophes. The IEDS living pod consists of a system of exterior and interior panels that inflate to cushion and protect the pod exterior against flying debris and its interior during relocation. The panels are connected to a series of internal air pumps that respond to disturbances in the natural environment and are activated when necessary to provide the appropriate level of defense. While the exterior and interior inflate to accommodate the influences of high winds or vigorous movement, the interior also may be inflated or deflated to accommodate different spatial demands. The pods are conceived without rigid foundations. Therefore, they migrate and their relocation is shaped by the dynamic forces of the wind and the configuration of the landscape. On one hand these nomadic, technologically equipped pods resist damage and on the other their movement creates migrating urban landscapes responsive to the weather conditions and shaped by its land configuration. The lack of permanency of their location suggests that these pods would eventually be brought to a locality that is less affected by the meteorological catastrophes.

The Urban Reef and Charged Landscapes projects explore productive relationship of an architectural intervention with the larger ecology. The Urban Reef project (Figure 5) by Caitlyn Browning is conceived as remediation of a heavily polluted industrial area in Detroit. It relies on synthetically produced protocells that use pollutants to produce matter that provides a new ground and building material for the site. The project is supported by current research in synthetic biology and a capability to produce synthetic "organisms" that could be programmed to consume specific substances. The protocells are distributed throughout the polluted terrain by following the topography and the level of pollution. The density and the distribution of the growth are channeled through an infrastructure that supports its hardening and is integrated into the topography of the site. The emerging landscape is a product of all those forces as they work across the site. The infrastructure distribution is related to the projected program that evolves over a long period of time. Nothing in this project is entirely predictable. The design of this environment is driven by the processes that will generate new ground. The program and activities on the site change over time based on the site's terrain transformation. The proposed infrastructure mediates and forms the terrain so that at different phases of its formation it could support specific activities.

The Charged Landscapes project (Figure 6) by Jose Trinidad takes advantage of under-utilized sites that intersect with highvoltage transmission routes passing through the city of Calgary. The project charts the network of electromagnetic energy and attempts to overlay new "metabolic morphologies" ${ }^{3}$ upon

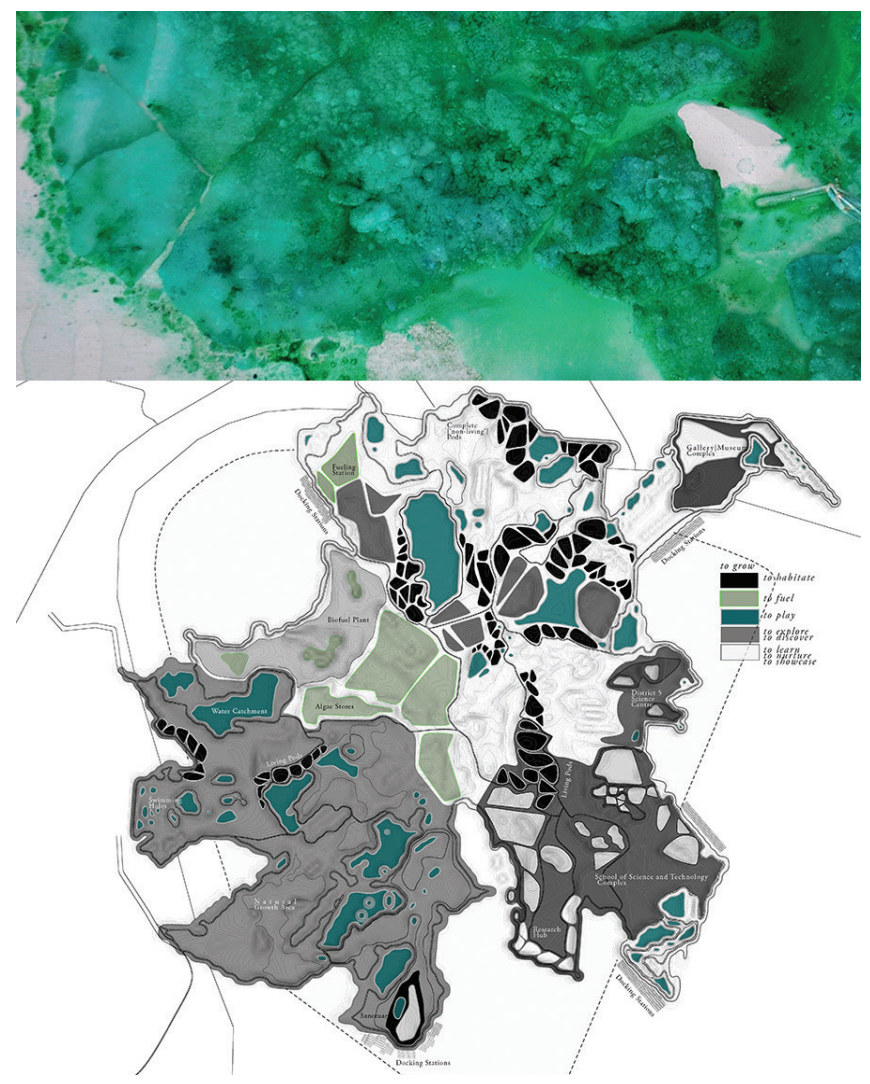

Figure 5 Above: The Urban Reef by Caitlyn Browning.

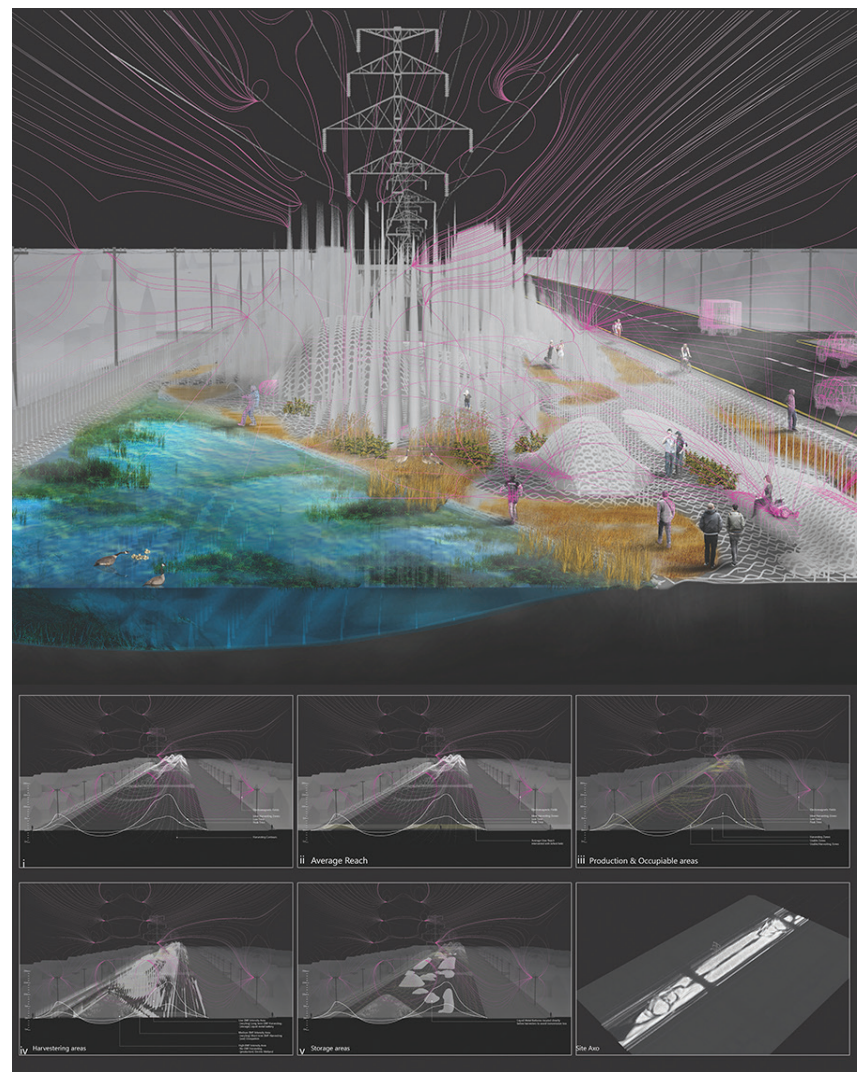

Figure 6: Charged Landscape by Jose Trinidad 
existing single-purpose energy infrastructures. Diagraming the form and flux of the electromagnetic boundaries around high voltage transmission lines reveals patterns of consumption, the potential of harvesting wasted residuals, and its hazards to health and the environment. Through this process, zones of energy production, storage, recycling, and human occupation are established. Together, these zones synthesize into productive, connective, and charged landscapes. The project attracts public interaction and participation by creating regions for human occupation that mediate the external environment by generating heat. Spaces delineated in this way are in constant flux; their sizes are determined by the interplay of released energy and air temperature. This is not a stable environment; to be in such a thermally modulated space one has to accept its fluctuating thresholds.

The Urban Reef and the Charged Landscape projects use different logics to construct drivers of the new environments. The Urban Reef uses bio-logic and strives to imprint that logic on the emerging new ground and unfolding of program over time, while the Charged Landscape uses energy distribution as an initial driver for patterns of energy harvesting and human occupation.

These projects tap into the idea of metabolism by exploring qualities that an artificial permeable boundary (supporting information of energy feedback loops) should have as an interface between the natural and the constructed. Michael Weinstock suggests that the notion of metabolism if linked to design could "relate pattern and process, form and behavior with spatial and cultural parameters"4 and support a symbiotic relationship of architecture with the natural world. In his seminal book, Evolutionary Architecture, John Frazer argues for a new form of designed artifact, one that is interacting and evolving in harmony with natural forces, including those of society. ${ }^{5}$

The idea of coupling the responsive, sensing or 'bio' technologies with the notion of metabolism opens the possibility of an intelligent, environmentally sensitive built environment that is connected to broader metabolic networks. Buildings that could sense and interact with its environment can operate more synergistically within larger ecologies and therefore can move closer to more sustainable participation within the global environment. The responsive architectural systems could act as ecologies in themselves, allowing architecture as a discipline to recalibrate its role in the larger socio-economic context by becoming a more intelligent and operative participant - a participant imbued with foresight.

\section{EXPANDING RESILIENCY}

In these projects the deployed active and sensing matter and material systems would result in a kinetic effect, a change of their configuration, or a capacity to grow or be generated.
Such general capacity for change suggests that regardless of technological or traditional practices towards resilience, what we need to do in these times of proliferating technologies and abundant disruptions is to be flexible and fluid with what arises. The forces that govern our ecologies, natural or engineered, are dynamic and changing. The technology alone will not save or emancipate humanity from the constraints of the turbulent environment, but it will enable us to extend more effectively into our environment and tap into constructive feedback loops of information or energy that would facilitate a more seamless transition between the constructed and the natural.

We extend ourselves into the environment with our technological artifacts. As we do that, the boundary between the internally developed and externally designed is constantly being contested. In this space, where the rules of the animate and inanimate, the living and synthetic overlap, 'designing' the resilience is a concept worth exploring. The prevailing mindset of the engineered might present a particular challenge in accepting the new forms of resiliency that tap into the indeterminate. For example, in engineering, specificity of a problem definition allows for an efficient solution. Operating in the space between the natural and the constructed might not always provide a context for a clear definition of the problem and a path to its solution. This space (in between) would require a transition, an interface, or a permeable boundary through which different logics could be brought into productive contact and generate new possibilities and realities. In other words, it requires fluid thresholds that can bridge the difference between the internal logic of the constructed and the logic of thermodynamic or indeterminate.

If we could through technological augmentation bring the constructed environment closer to the resiliency of natural ecologies we might be able to mediate consequences of sudden or persistent exigencies. In his text "Resilience and Stability of Ecological Systems" C.S. Holling talks about the change of a domain in ecological systems - a situation when permanent change takes hold within the system and influences the system towards a change. An example of this is an invasion and establishment of shrubs and trees in cattle grazing areas. When they gain sufficient density and cattle moves elsewhere the grassland will not reestablish itself. Only if trees and shrubs are removed grassland can return. An interesting point he makes is that in natural systems the question is not how stable they are but how likely they are to change domains and stay in the changed configuration. ${ }^{6}$ As C.S. Holling suggests, natural systems that are constantly confronted with unpredictable internal and external changes are less concerned with constancy and more with persistence of the relationships. ${ }^{7}$ On the other hand, engineered systems 
or devices that perform specific tasks under predicable external conditions have their performance goal immediately adjusted if the variation in performance is observed; they are concerned with constancy of performance. An equilibriumcentered view of such systems is static and doesn't support transient behavior of natural systems. Awareness of this distinction could be influential when designing within a dynamic design space in which the natural and constructed meet.

When designing active and adaptive artificial environments, whether they are intelligent facades or built environments that connect to natural ecologies, it would be useful to establish relationships that persist between the natural and constructed so that, when interfaced, they could behave similarly. In this context we might be less interested in stability of an engineered ecology and more in the zones of stability and establishing its gradient (or ability to perform under the constant change). Analogous to Holling's view, the boundaries or limits of such ecologies might facilitate change of the domain. ${ }^{8}$ Designing such indeterminate systems would certainly require a change in design attitudes.

Furthermore, a built environment is deemed resilient if there is a coordinated effort, as in the case of a natural disaster that would alleviate the effects of a disaster and foster a fast return to the normalcy. Such a focus on the return to a pre-disaster condition of the environment (through fast rebuilding, for example) doesn't account for emergence of different conditions and configurations of that environment that might call for some built-in behaviours (of the built environment) that would allow its re-configuration.

Thinking in terms of exchange, dynamics, energy, and flow and not in terms of assembled elements affects the way we think about architecture. It fosters thinking in terms of thresholds and not of constructed impermeable boundaries. Thresholds support the notions of gradients and transitions. Thresholds, gradients and transitions are qualities of ecological resiliency. If we succeed in supporting these conditions through technological augmentation of the constructed environment we should be able to disperse technologies that could be activated locally and only when needed. For example, instead of heating or cooling an entire building, the heat or coolness effect could 'travel' with the occupant. Reyner Banham reminds us that two basic ways of controlling environment were by hiding under the tree/tent/roof (in other words, by building a shelter) or by mediating local environment by campfire. He points out that "a campfire has many unique qualities which architecture cannot hope to equal, above all, its freedom and variability." ${ }^{\prime \prime}$ It was his argument for the inclusion of environmental phenomena and their variability into a design process that began to orient architecture towards adaptive environments.
The examples where a boundary between external and internal condition, or the variability of a phenomena is used as a driver for design can be found in the work of Philippe Rahm and Sean Lally. For them, the essence of architecture is to create a gradient of temperature, humidity, air or sound that provides fluid, dissolved boundaries of comfortable or desired conditions (the atmospheres, as articulated by Rahm). Inhabitation of these spaces is driven by a desire or need for a particular sensorial experience or comfort. Architecture's agenda in these projects, similar to Reyner Banham's ideas, encompasses the domain of environment and not that of the object. If we could construct atmospheres by modulating flows of heat, coolness, air or noise, the boundary is then dissolved into phenomena that exert subtle influence and support organization of people and activity differently than physical boundaries would. Such design logic would begin to erode traditional notions of control and organization of space and 'contaminate' design with the notion of variability, leading to a richer interaction with the built environment. For example, if the infrastructure for space organization is not concerned with the traditional logic of the constructed but is informed by the logics of thermodynamic behaviours, that would lead to new notions of order and organization of space. Such an attitude, however, would increase organizational complexity, introduce emergence, resulting in the design of open systems. ${ }^{10}$

Rahm's architectural projects invite us to re-think the wall as an impermeable tectonic element that separates interior and exterior by introducing a concept of the wall as strata. For example, to insure good thermal performance of buildings we often add insulation. Rahm proposes that instead of adding thermal layers to the wall we should stratify (programmatic) spaces as different thermal zones, organizing a building program in terms of their thermal coefficients, thus triggering thermodynamics of the airflow by using thermal difference between the spaces. The space with the lowest coefficient would be at the center and those with the highest coefficient on the periphery; the thermal difference would trigger a constant flow of air, forming an internal climate of gradients. In this way, the boundaries between inside and outside would dissolve into different spatial and thermal thresholds. Such an approach requires a different logic for space organization based on thermal zones and air flows. The built environment can be organized by defining zones of transition, comfort, economy, transportation ... and, by aligning itself with a larger environment, could result in stratification and re-layering of its regions and their appropriation through occupation and use as a strategy for bringing the constructed and natural together.

Recent research advances in the field of synthetic biology offer another promising direction for changing the way in which buildings participate in their environment. In synthetically produced biological materials, "intelligence" is embedded in 
the matter itself. In 1974 Waclaw Szybalski suggested that synthetic biology "would be a field with an unlimited expansion potential and hardly any limitations to building 'new better control circuits' or [...] finally other 'synthetic' organisms." ${ }^{11}$ In an article published in 2010, Rachel Armstrong discusses a new class of materials, developed with technologies derived from synthetic biology, which are capable of 'decision making' by relying on the chemical computational power of their molecules. ${ }^{12}$ They are 'programed/designed' to make decisions about their environment and respond to it in complex ways that involve a change in their form, function or appearance. Responsiveness of these materials lies in their capacity for chemical computation. Without the need to rely on traditional computing methods and actuation devices, these materials offer a very different way of imagining an operational capacity of matter. ${ }^{13}$ Armstrong's Living Brick project puts these ideas to work by proposing a brick based on Microbial Fuel Cell (MFC). These metabolically active building blocks can harness the metabolic power of microbes and convert it into electricity. ${ }^{14}$ Structures made of this material can become active contributors to their environment and even appropriated by living creatures or other substances or chemicals. As such, they can choreograph behaviors or transformations of their physical or chemical context.

\section{CONCLUSION}

As we move from an attitude of determinacy and constancy (in engineering systems) towards the one of indeterminacy and adaptability, we move closer towards resiliency. Furthermore, the disruption of resilient systems results in their transformation over time. Designing change over time in the built environment, however, is a challenging proposition that can change the conceptual basis of design. It means that instead of designing for a particular condition and under specific design programs or criteria, we would design for change and transformation, taking into account the time that is necessary for a particular change to happen (as in the Urban Reef project). In such a 'mutable' design process, the design elements might include designed disruptors that would act as catalysts (as in the Charged Landscapes of Swarm Spaces projects).

Natural systems are open systems in which invasion and reinvasion of disruptors is a pivotal factor; they are constantly in transient state. ${ }^{15}$ If we need to interface with them through technology, these new synthetic systems should be sensitive and responsive to natural system and its fluctuations (as in the Swarm Spaces and Remedia[c]tion projects). Striving for consistency of performance might move the system away from resiliency. Instead, we should strive for designing our constructed environment as an open system and accept transformation over time as an integral part of the design process and its life.
To form an interface or a permeable boundary between the designed and its local ecology requires a redefinition of the design framework to include fluidity of processes. Ibanez and Katsikis call this expanded framework the Grounding Metabolism and base it on a concept of urban metabolism. ${ }^{16}$ The porosity of boundaries and a blurred distinction between the natural and constructed (or the natural and social as Jason Moore would suggest) allows design to expand its territory. ${ }^{17}$ It makes the struggle to engage with the larger context less challenging by undermining the distinctions. The shift from a binary relationship to the recognition of interdependencies enables designers to focus on the interactions between social and ecological processes, which in turn makes conditions produced by these interactions more visible. The dynamics of the interactions would move environmental factors from the insulated position of an add-on factor to an equal participant as "producer and product of the web of life"18, potentially shifting the design space towards awareness of a constant production of new conditions and a new ground. ${ }^{19}$ As Ibanez and Karsikis suggest, the temptation to engage larger territories comes from increasingly complex urban environments and also from the need to understand that complexity and its interdependencies and influences on the social and ecological networks across the planet. ${ }^{20}$

If we were to accept change as a fundamental contextual condition, architecture could then begin to truly mediate between the built environment, the people who occupy it and the larger context. As Ed van Hinte notes, "instead of being merely the producer of a unique three-dimensional product, architects should see themselves as programmers of a process of spatial change." ${ }^{\prime 21}$ The principal task for architects is to create "a field of change and modification" that would generate possibilities instead of fixed conditions. ${ }^{22}$

To be resilient architecture has to form dynamic relationships to the external environment. It should interact with various conditions regardless of their nature (favorable, benign or polluting). In such a context, architecture is no longer a controlling agent sealing off its occupants from the surroundings but an agent of dynamic exchange between the interior and exterior. A porous relationship between the interior and exterior is what could promote that dynamic relationship. We should suspend a challenge of seeking a non-permeable and clearly defined boundary between inside and outside, the constructed and the natural, and instead design open systems that foster a constant flow of information, matter and energy. 


\section{ENDNOTES}

1. Frei Otto, Pneu and Bone, (Stuttgart: Institute for Light Structures, 1995), 8.

2. James Graham Ballard, "The Thousand Dreams of Stellavista" in Vermilion Sands, (London: Vintage, 2001), 185-208.

3. Michael Weinstock, "Metabolism and Morphology," Architectural Design, March/April (2008): 26-33.

4. Ibid.

5. Frazer, John, An Evolutionary Architecture (London: Architectural Association, 1995)

6. C.S. Hoiling, "Resilience And Stability Of Ecological Systems," Annual Review of Ecology and Systematics 4, (1973): 1-23.

7. Ibid.

8. Ibid., 10

9. Reyner Banham, "A Home is not a House," Art in America 2, April (1965): 75.

10. Alan Pavé, "Biological and Ecological Systems Hierarchical Organization", in Hierarchy in Natural and Social Sciences, ed. Denise Pumain, (New York: Springer-Verlag, 2006), 39-70.

11. Szybalski, Wacław, "In Vivo and in Vitro Initiation of Transcription" in Control of Gene Expression, ed. Alexander Kohn and Adam Shatkay, (New York: Plenum Press, 1974): 23.

12. Armstrong, Rachel, "Systems Architecture: A New Model for Sustainability and the Built Environment using Nanotechnology, Biotechnology, Information Technology, and Cognitive Science with Living Technology", in Artificial Life 16, no.1 (2010): 73-87.

13. Ibid.

14. Tafline Laylin, "'Living Bricks'” could turn sunlight and waste water into energy-generating bioreactor walls," Inhabitat, https://inhabitat. com/living-bricks-could-turn-sunlight-and-waste-water-into-energygenerating-bioreactor-walls/

15. C.S.Holling, Resilience And Stability Of Ecological Systems, 1-23

16. Daniel Ibañez and Nikos Katsikis, "Grounding Metabolism," New Geographies 06 (2014): 3.

17. Jason W. Moore, "Towards a Singular Metabolism," New Geographies 06 (2014): 10-19.

18. Ibid., 12

19. Ibid., 14

20. Ibañez and Katsikis, Grounding Metabolism, 3.

21. Ed van Hinte, Marc Neelen, Jacques Vink, and Piet Vollaard, Smart Architecture (Amsterdam: 010 Publishers, 2003), 132

22. Ibid., 132. 